\title{
Particle Impact Energy Variation with the Size and Number of Particles in a Planetary Ball Mill
}

\author{
Fumie HIROSAWA, Tomohiro IWASAKI*, and Masashi IWATA \\ Department of Chemical Engineering, Osaka Prefecture University, 1-1 Gakuen-cho, Naka-ku, Sakai, Osaka 599-8531, Japan
}

\begin{abstract}
To investigate the mechanical energy applying to the particles in a grinding process using a planetary ball mill, the impact energy of particles was estimated by simulating the behavior of the particles and grinding balls using the discrete element method (DEM) under different conditions of the size and number of particles, corresponding to their variations during milling. As the impact energy contributing to the particle breakage, we focused on the particle impact energy generated at particle-to-grinding ball/wall and particle-to-particle collisions. The particle size and the number of particles affected the level of particle impact energy at a single collision and the number of collisions of particles, respectively, resulting in an increase of the total impact energy of particles with decreasing particle size and increasing number of particles. The result suggests that milling conditions such as the size of grinding balls should be adjusted appropriately based on the variation of the size and number of particles so that the particles can receive large amounts of the impact energy during milling.
\end{abstract}

\section{Introduction}

Dry powder grinding is an important unit operation in many industries, such as mining, food, fine chemical and pharmaceutical, ranging from coarse mineral ore to submicrometer-sized fine drug powder. Recently, ultrafine dry grinding processes with high energy milling (Chen et al., 2015; Guzzo et al., 2015; Kleiv and Thornhill, 2007; Guzzo et al., 2019), which can produce fine particles with improved properties and/or enhanced performance, have attracted much attention. In ultrafine dry grinding processes, planetary ball mills have often been employed since the particles receive remarkably high impact energy at collisions with the grinding balls and the mill pot wall. The impact energy can vary depending strongly on the milling conditions and lead to breakage of particles, resulting in decreasing the particle size and increasing the number of particles. Therefore, the impact energy alters the produced particle properties, such as size distribution, specific surface area and microscopic structure. Accordingly, for producing the particles with controlled properties, the impact energy must be appropriately adjusted.

In the ultrafine dry grinding, due to the high impact energy applied to the particles, the particle size and number of particles drastically varies during milling (Kleiv and Thornhill, 2007; Chen et al., 2015; Guzzo et al., 2015; Guzzo et al., 2019), which can change the type and amount of the impact energy of particles. Therefore, as a fundamental study for adjusting the impact energy, it is necessary to investigate the variation of the impact energy with the size and number of particles.
In some studies, variations of the impact energy with the revolution speed of the mill pot (Minagawa et al., 2018) and the loading masses of the particles and grinding balls (Ashrafizadeh and Ashrafizaadeh, 2012; Geissbuhler and Sawley, 2013) were obtained using the discrete element method (DEM) simulation of behavior of the particles and balls. However, to the best of our knowledge, no studies have focused on the impact energy variation with the size and number of particles.

In this paper, in order to provide criteria for adjusting the impact energy in accordance with the progress of particle breakage, we estimated the impact energy by simulating the behavior of the particles and grinding balls in a planetary mill under different conditions of the size and number of particles using DEM. To study in detail the effects of the size and number of particles on the impact energy, the impact energy was analyzed separately by classifying the impact energy of a particle into two collision categories, i.e., particle-to-particle and particle-to-grinding ball/wall collisions.

\section{Methods}

To obtain the impact energy of the particles, the behavior of the particles and grinding balls in a planetary mill under dry conditions was simulated (Hirosawa et al., 2019) using DEM (Cundall and Strack, 1979), which is a reliable method for simulations of solid particle behaviors (Horabik and Molenda, 2016; Tang et al., 2018). In this work, the simulation method proposed by Tsuji et al. (1992) was employed. The simulation

*Corresponding author: iwasaki@chemeng.osakafu-u.ac.jp 
parameters, such as the density, Poisson's ratio, Young's modulus of particle, ball and wall materials, and the restitution coefficient and friction coefficient of particleto-particle, particle-to-ball/wall and ball-to-ball/wall collisions, were the same as those used by Capece et al. (2014), as listed in Table 1. In order to obtain the variation of the impact energy of particles with respect to the particle breakage, both the particle size and the number of particles were changed independently as shown in Table 2 . The variation of the size and number of particles was expressed by a diameter ratio $D_{\mathrm{b}} / D_{\mathrm{p}}$, where $D_{\mathrm{b}}$ and $D_{\mathrm{p}}$ are the diameters of a ball and a particle, respectively. The ball-to-particle filling mass ratio (BPR) was kept at 30 regardless of the size and number of particles.

In order to evaluate the energy spent for the particle breakage by impact milling, the impact energy $E$ of particles is defined by the energy dissipated through a particle collision, according to Govender et al. (2015), Santhanam and Dreizin (2012) and Cleary and Morrison (2011). The impact energy is calculated by integrating the normal and tangential contact forces, $F_{\mathrm{n}}$ and $F_{\mathrm{t}}$, of a particle with respect to its overlaps, $\delta_{\mathrm{n}}$ and $\delta_{\mathrm{t}}$, over the contact period $t_{\mathrm{c}}$ in the case of no sliding at the contact, as expressed in Eq. (1).

$$
E=\int_{0}^{t_{\mathrm{c}}}\left(F_{\mathrm{n}} \mathrm{d} \delta_{\mathrm{n}}+F_{\mathrm{t}} \mathrm{d} \delta_{\mathrm{t}}\right)
$$

Table 1. Parameters used in the simulation

\begin{tabular}{ll}
\hline Number: & 18 \\
Grinding ball & $18-3724$ \\
Particle & 30 \\
Ball/particle filling mass ratio & 16 \\
Ball/particle filling volume ratio & \\
Diameter: & $10.0 \mathrm{~mm}$ \\
Grinding ball & $4.0-0.67 \mathrm{~mm}$ \\
Particle & \\
Density*: & $4000 \mathrm{~kg} / \mathrm{m}^{3}$ \\
Grinding ball & $2150 \mathrm{~kg} / \mathrm{m}^{3}$ \\
Particle & 0.3 \\
Poisson's ratio* & $1 \times 10^{7} \mathrm{~Pa}$ \\
Young's modulus* & 0.75 \\
Coefficient of restitution* & 0.75 \\
Sliding friction coefficient* & 0.02 \\
Rolling friction coefficient* & $45 \mathrm{~mL}$ \\
Pot volume & $40 \mathrm{~mm}$ \\
Pot diameter & $35.8 \mathrm{~mm}$ \\
Pot depth & $10.0 \mathrm{~s}^{-1}$ \\
Revolution speed & $67 \mathrm{~mm}$ \\
Revolution radius & -1 \\
Rotation-to-revolution speed ratio & $200 \mathrm{~ns}$ \\
Time step & \\
\hline
\end{tabular}

* Capece et al. (2014)
Table 2. Particle sizes and numbers of particles in the simulation

\begin{tabular}{ccc}
\hline $\begin{array}{c}\text { Particle size, } \\
D_{\mathrm{p}}[\mathrm{mm}]\end{array}$ & $\begin{array}{c}\text { Number of } \\
\text { particles }[-]\end{array}$ & $D_{\mathrm{b}} / D_{\mathrm{p}}[-]$ \\
\hline 4.0 & 18 & 2.5 \\
2.7 & 59 & 3.75 \\
2.0 & 140 & 5 \\
1.6 & 273 & 6.25 \\
1.3 & 476 & 7.5 \\
1.0 & 1120 & 10 \\
0.80 & 2188 & 12.5 \\
0.67 & 3724 & 15 \\
\hline$D_{\mathrm{b}}=$ Ball size $(10 \mathrm{~mm})$ &
\end{tabular}

When the contacting particle slides, the impact energy $E$ in the tangential direction is calculated by integrating the product of the contact force $F_{\mathrm{t}}$, i.e., the frictional force, and the tangential velocity $v_{\mathrm{t}}$, over the contact period $t_{\mathrm{c}}$.

$$
E=\int_{0}^{t_{\mathrm{c}}}\left(F_{\mathrm{n}} \mathrm{d} \delta_{\mathrm{n}}+F_{\mathrm{t}} v_{\mathrm{t}}\right)
$$

The total impact energy $E_{\mathrm{t}}$ was obtained by summing up $E$ for all collisions of the particles. Additionally, the total particle impact energies, $E_{\mathrm{tb}}$ and $E_{\mathrm{tp}}$, at the particle-toball/wall collisions and the particle-to-particle collisions, respectively, were also investigated, of which the relationship was expressed by Eq. (3).

$$
E_{\mathrm{t}}=E_{\mathrm{tb}}+E_{\mathrm{tp}}
$$

\section{Results and Discussion}

Figure 1 illustrates the snapshots of the particles and grinding balls in the mill pot at different $D_{\mathrm{b}} / D_{\mathrm{p}}$ values corresponding to different stages of the particle breakage. Regardless of $D_{\mathrm{b}} / D_{\mathrm{p}}$, the balls were packed densely near the wall of mill pot because of high centrifugal accelerations induced by the planetary motion of the mill pot. Smaller particles also tended to gather near the wall. Therefore, the smaller particles may receive large amounts of the impact energy at particle-to-ball/wall collisions near the wall.

Figure 2 shows the impact energy distributions of the particles at different $D_{\mathrm{b}} / D_{\mathrm{p}}$ values. Smaller impact energies increased at high values of $D_{\mathrm{b}} / D_{\mathrm{p}}$. The energy distributions, except for a case at $D_{\mathrm{b}} / D_{\mathrm{p}}=2.5$, were approximately bimodal, consisting of high and low energies. The higher and lower impact energies could be generated at particle-to-ball/wall and particle-to-particle collisions, respectively. At all $D_{\mathrm{b}} / D_{\mathrm{p}}$ values, the most of the impact energy generated at the particle-to-ball/wall collisions exceeded a threshold energy of the particle breakage (Capece et al., 2014), determined by the material and size of the particles. On the other hand, more than half of the impact energy generated at the particle-to-particle collisions was lower than the threshold energy. Therefore, the particle breakage may strongly depend on the collision types of the particles determining the level of impact energy. 

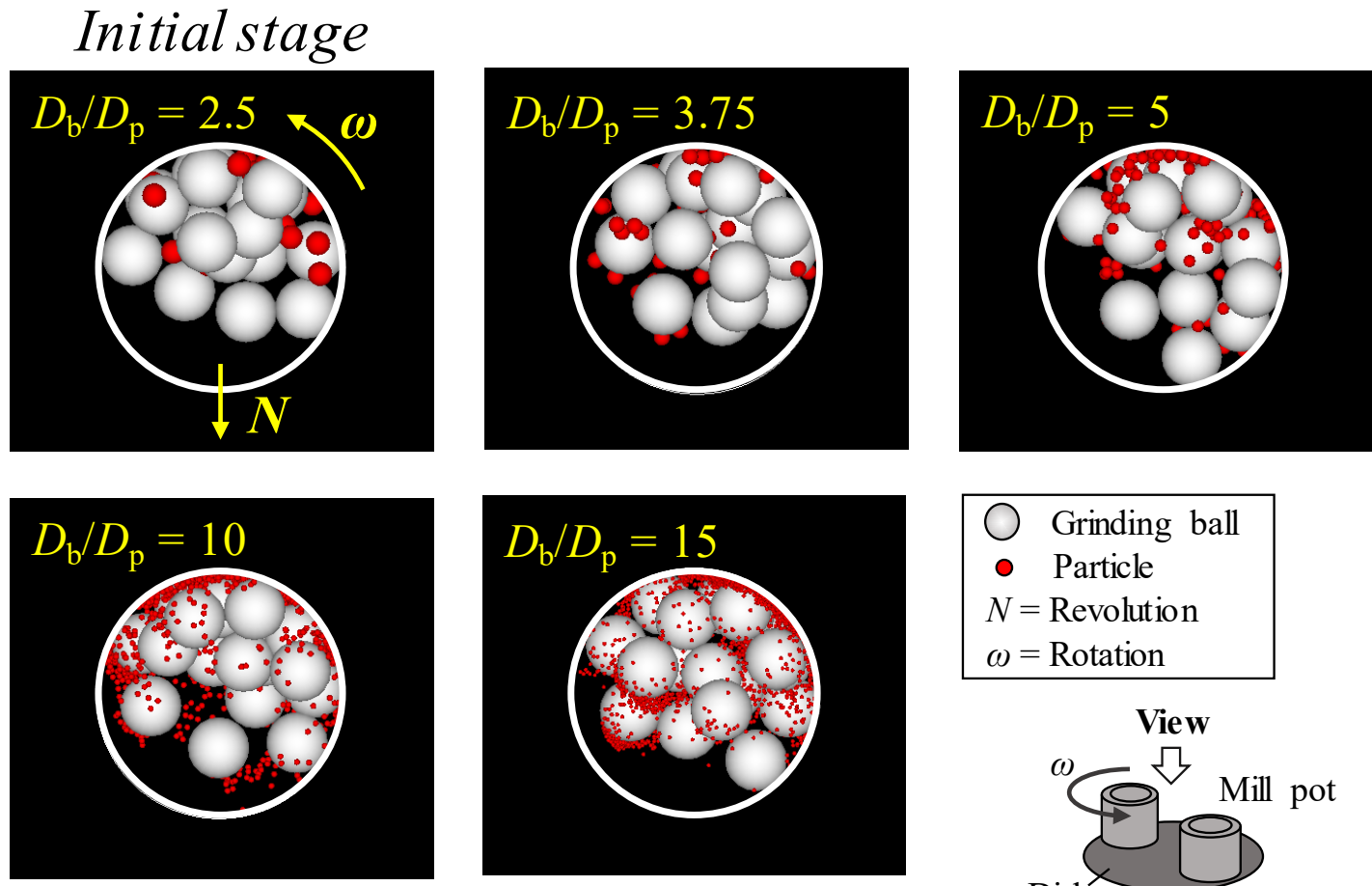

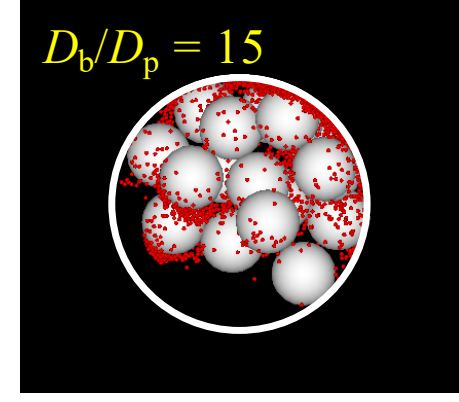

Final stage
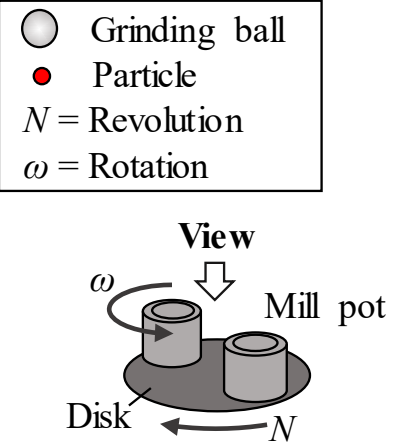

Figure 1. Snapshots of particles and grinding balls in planetary mill at different stages of particle breakage

In order to investigate the effect of collision types on the impact energy variations with the size and number of the particles, we calculated the impact energy separately at the particle-to-ball/wall and particle-to-particle collisions. The effect of the particle size on the impact energy of particles was expressed by a median impact energy corresponding to the $50 \%$ value of the accumulated impact energy representing a representative of the impact energy at a single collision. Figure 3 shows the change in the median impact energy with the mass of a single particle at each collision type. The median impact energy at the particle-to-ball/wall collisions was almost constant regardless of the mass of a particle and much higher than that at the particle-to-particle collisions, showing that the impact energy at the particleto-ball/wall collisions can greatly affect the particle breakage. However, at the particle-to-particle collisions, the median impact energy showed a tendency to remarkably increase with increasing mass of a particle in comparison with that at the particle-to-ball/wall collisions. Subsequently, the effect of the number of particles on the impact energy at each collision type was studied based on the number of collisions of particles, as shown in Figure 4. The numbers of particle-to-ball/wall collisions increased linearly with increasing number of particles. On the other hand, at the particle-to-particle collisions, the number of the collisions exponentially increased due to the drastic increase in the number of particles.
For investigating the amount of impact energy applied to the particles during milling, the total impact energies, $E_{\mathrm{t}}, E_{\mathrm{tb}}$ and $E_{\mathrm{tp}}$, were calculated, as illustrated in Figure 5. The total impact energies increased with increasing $D_{\mathrm{b}} / D_{\mathrm{p}}$ values, i.e., decreasing particle size and increasing number of particles simultaneously at a constant filling mass of the particles, because the number of particles greatly affected on the impact energy in comparison with the particle size. $E_{\mathrm{tb}}$ was remarkably higher compared with $E_{\mathrm{tp}}$, and close to $E_{\mathrm{t}}$ at low $D_{\mathrm{b}} / D_{\mathrm{p}}$ values. However, the influence of $E_{\mathrm{tp}}$ on $E_{\mathrm{t}}$ gradually increased with increasing $D_{\mathrm{b}} / D_{\mathrm{p}}$, implying that the amount of impact energy contributing to the particle breakage may relatively decrease at extremely high $D_{\mathrm{b}} / D_{\mathrm{p}}$ values.

\section{Conclusion}

The variation of particle impact energy with the particle size and number of particles in a planetary ball mill was investigated by simulating the behavior of the particles and grinding balls using DEM. The median impact energy at a single collision decreased with reduction of the particle size. However, the number of collisions of the particles drastically increased with an increase in the number of particles. Comparing to the particle size reduction, the increase of the number of particles greatly affected the particle impact energy, resulting in an 


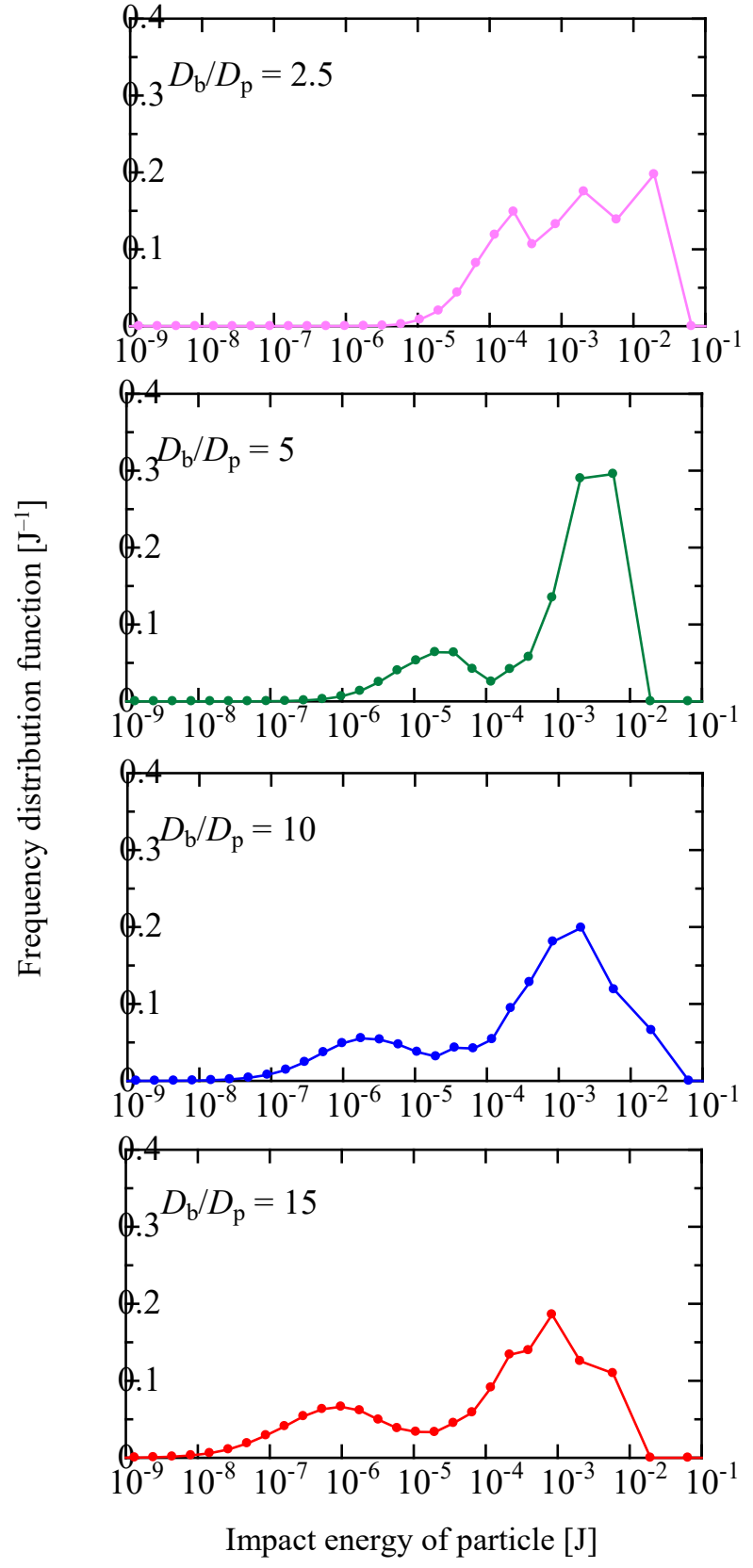

Figure 2. Effect of $D_{\mathrm{b}} / D_{\mathrm{p}}$ on impact energy distribution of particles

increase of the total impact energy contributing to the particle breakage. The result suggests that the size of grinding balls should be adjusted appropriately based on the variation of the size and number of particles so that the particles can receive large amounts of the impact energy during milling.

\section{Acknowledgement}

This work was supported by JSPS Research Fellowships for Young Scientists, and JSPS KAKENHI Grant Number JP18J21860.

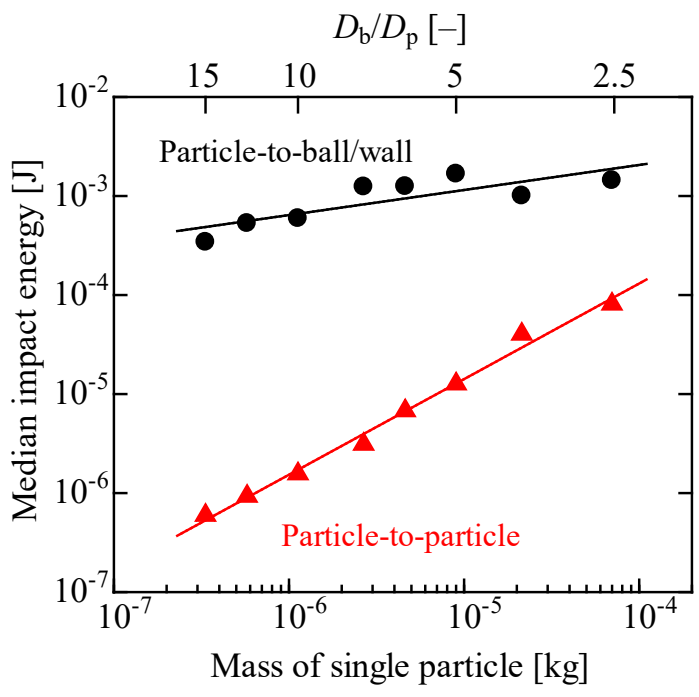

Figure 3. Effect of collision types on median impact energy variation with mass of single particle.

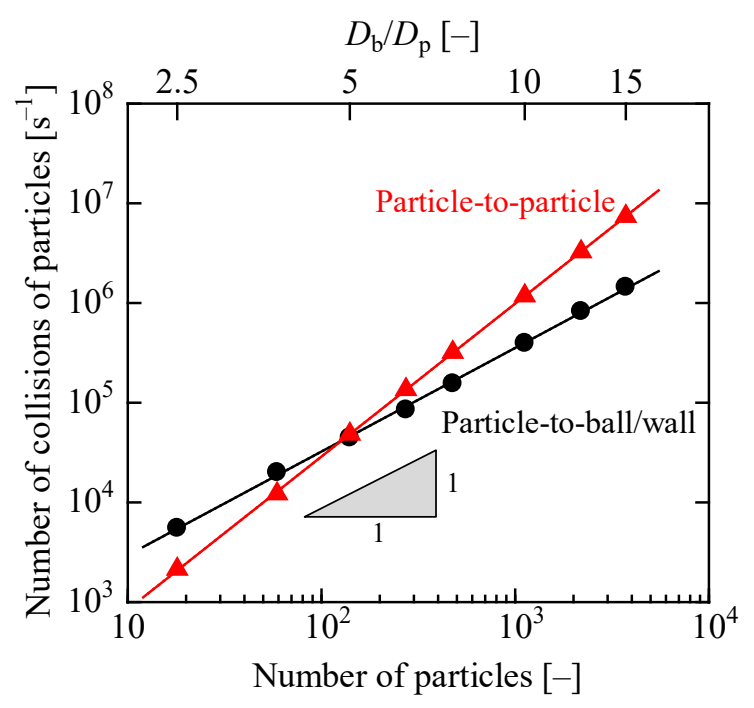

Figure 4. Effect of collision types on change in number of collisions with number of particles

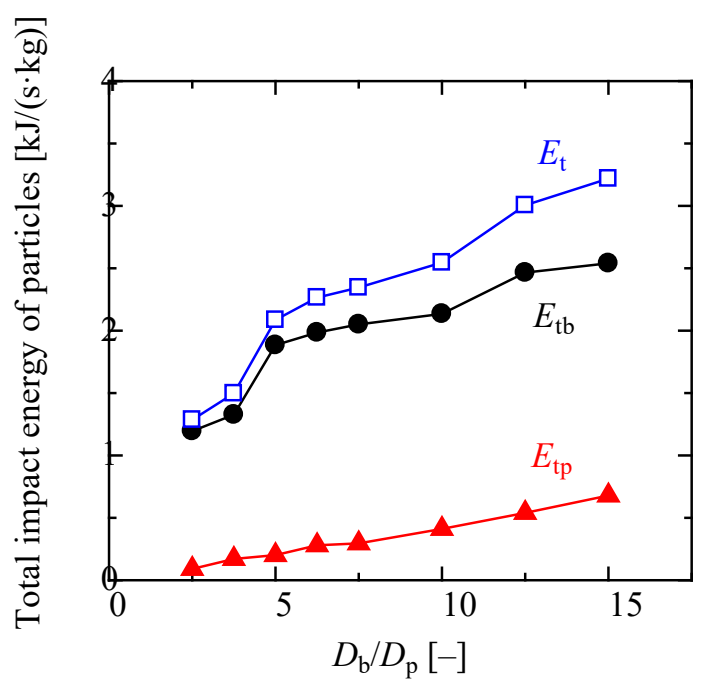

Figure 5. Effect of collision types on total impact energy variation with $D_{\mathrm{b}} / D_{\mathrm{p}}$ 


\section{References}

Ashrafizadeh, H. and M. Ashrafizaadeh; "Influence of Processing Parameters on Grinding Mechanism in Planetary Mill by Employing Discrete Element Method," Adv. Powder Technol., 23, 708-716 (2012)

Capece, M., E. Bilgili, and R. Davé; "Insight into Firstorder Breakage Kinetics Using a Particle-scale Breakage Rate Constant," Chem. Eng. Sci., 117, 318-330 (2014)

Chen, Y., X. Lian, Z. Li, S. Zheng, and Z. Wang; "Effects of Rotation Speed and Media Density on Particle Size Distribution and Structure of Ground Calcium Carbonate in a Planetary Ball Mill," $A d v$. Powder Technol., 26, 505-510 (2015)

Cleary, P. W. and R. D. Morrison; "Understanding Fine Ore Breakage in a Laboratory Scale Ball Mill Using DEM," Miner. Eng., 24, 352-366 (2011)

Cundall, P. A. and O. D. L. Strack; "A Discrete Numerical Model for Granular Assemblies," Géotechnique, 29, 47-65 (1979)

Geissbuhler, D. and M. L. Sawley; "Particle Motion and Energy Dissipation Spectra in a Planetary Ball Mill," III International Conference on Particle-based MethodsFundamentals and Applications PARTICLES 2013, pp. 236-246, Stuttgart, Germany (2013)

Govender, N., R. K. Rajamani, S. Kok, and D. N. Wilke; "Discrete Element Simulation of Mill Charge in 3D Using the BLAZE-DEM GPU Framework," Miner. Eng., 79, 152-168 (2015)

Guzzo, P. L., A. A. A. Tino, and J. B. Santos; "The Onset of Particle Agglomeration during the Dry Ultrafine Grinding of Limestone in a Planetary Ball Mill," Powder Technol., 284, 122-129 (2015)

Guzzo, P. L., F. B. Marinho de Barros, and A. A. A. Tino; "Effect of Prolonged Dry Grinding on Size Distribution, Crystal Structure and Thermal Decomposition of Ultrafine Particles of Dolostone," Powder Technol., 342, 141-148 (2019)

Hirosawa, F., T. Iwasaki, and M. Iwata; "Kinetic Analysis of Mechanochemical Reaction between Zinc Oxide and Gamma Ferric Oxide Based on the Impact Energy and Collision Frequency of Particles," Powder Technol., 352, 360-368 (2019)

Horabik, J. and M. Molenda; "Parameters and Contact Models for DEM Simulations of Agricultural Granular Materials: A Review," Biosyst. Eng., 147, 206-225 (2016)

Kleiv, R. A. and M. Thornhill; "Production of Mechanically Activated Rock Flour Fertilizer by High
Intensive Ultrafine Grinding," Miner. Eng., 20, 334-341 (2007)

Minagawa, M., S. Hisatomi, T. Kato, G. Granata, and C. Tokoro; "Enhancement of Copper Dissolution by Mechanochemical Activation of Copper Ores: Correlation between Leaching Experiments and DEM Simulations," Adv. Powder Technol., 29, 471-478 (2018)

Santhanam, P. R. and E. L. Dreizin; "Predicting Conditions for Scaled-up Manufacturing of Materials Prepared by Ball Milling," Powder Technol., 221, 403$411(2012)$

Tang, J., J. Qiao, Z. Liu, X. Zhou, G. Yu, and J. Zhao; "Mechanism Characteristic Analysis and Soft Measuring Method Review for Ball Mill Load Based on Mechanical Vibration and Acoustic Signals in the Grinding Process," Miner. Eng., 128, 294-311 (2018)

Tsuji, Y., T. Tanaka, and T. Ishida; "Lagrangian Numerical Simulation of Plug Flow of Cohesionless Particles in a Horizontal Pipe," Powder Technol., 71, 239-250 (1992) 